
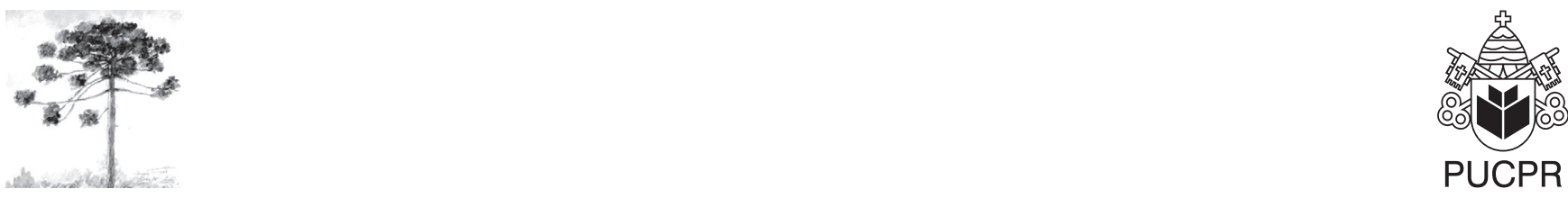

PUCPR

\title{
Metodologias de amostragem em macrófitas e seu efeito na abundância de odonata
}

\author{
A comparison of two sampling techniques in the study of the \\ macrophytes and their effects on abundance of odonata
}

\section{João Ânderson Fulan}

Doutorado (andamento), Departamento de Zoologia, Instituto de Biociências, Universidade Estadual de São Paulo (UNESP), Botucatu, SP - Brasil, e-mail: joaofulan@ig.com.br

\section{Resumo}

O objetivo deste trabalho foi comparar a abundância de Odonata em duas espécies de macrófitas, Eichhornia azurea e Salvinia auriculata, utilizando metodologias de amostragem distintas, puçá e quadrado vazado, em duas lagoas laterais ao Rio Paranapanema, SP. Três bancos de E. azurea e três de S. auriculata foram selecionados em cada lagoa em julho de 2003. Foram realizadas três réplicas em cada estação de amostragem para as macrófitas e para os fatores abióticos. Dentre os fatores abióticos, foram medidos temperatura de superfície da água, oxigênio, $\mathrm{pH}$ e condutividade. Foi realizado o teste de Student a fim de se comparar a média das abundâncias entre o puçá e o quadrado vazado e também uma análise de correlação entre as abundâncias de Odonata e fatores abióticos. Os resultados mostraram que o puçá e o quadrado vazado não diferem significativamente quanto às abundâncias. Entretanto, o puçá apresentou maior abundância total em todos os sítios analisados e mostrou-se mais eficiente na captura de ninfas menores como Telebasis sp., Tauriphila sp. e Erythemis sp.

Palavras-chave: Odonata. Macrófitas. Amostragem.

\section{Abstract}

The aim of this work was assess the distribution of the abundance of Odonata on macrophytes utilizing distinct methods sampling insect net of 0,25 mm mesh size and square method. The samplings were realized in two lateral 
lakes in its mouth zone into the Jurumirim Reservoir, São Paulo State, Brazil. Three stands of E. azurea and three of $\mathrm{S}$. auriculata were assessed. In each sampling station were measured surface temperature, dissolved oxygen, $p H$ and conductivity. It was realized two statistical analysis: the Student test to compare the abundances of Odonate with insect net and square and correlation between the abundances of Odonata and abiotic factors. The results showed that the abundances of Odonata did not differ significantly between insect net and square, however, insect net exhibited higher abundance in every one sites of sampling. Insect net also was efficient in the caught of small nymphs as Telebasis $s p$., Tauriphila $s p . e$ Erythemis $s p$.

Keywords: Odonata. Macrophytes. Sampling.

\section{Introduçáo}

Macrófitas são plantas que habitam desde brejos até ambientes verdadeiramente aquáticos como rios e principalmente lagos (1). Dentre os papéis desempenhados pelas plantas aquáticas, segundo o autor, podemos destacar a reciclagem de nutrientes do sedimento para a coluna de água pelo contato da raiz, substrato para comunidades fitoplanctônicas e refúgio contra predadores. Os refúgios são os principais fatores explicativos para a alta abundância de macroinvertebrados registrada entre as macrófitas (2). Odonata se destaca dentre os macroinvertebrados como um dos principais insetos predadores das zonas litorâneas (3). Segundo Corbet (4), as ninfas de Odonata possuem uma dieta extremamente generalista e se alimentam da maioria dos macroinvertebrados e até mesmo de vertebrados, como larvas de anfíbios e peixes, desde que possuam tamanho adequado. Apesar das ninfas de Odonata não serem o grupo mais abundante entre as macrófitas, representam uma das principais biomassas nas zonas litorâneas e desta forma desempenham importante papel na cadeia trófica como predadoras e presas e, por esta razão, merecem estudos apropriados (5).

Segundo Esteves (1), as plantas aquáticas foram as comunidades mais negligenciadas no sentido da falta de estudo e interesse por parte de pesquisadores nos últimos anos. Segundo o autor, a falta de padronização na metodologia de amostragem das macrófitas é a responsável pelos poucos trabalhos publicados. Estudos envolvendo Eichhornia azurea (Kunth), por exemplo, possuem metodologias de amostragem totalmente distintas, em que se define o espaço amostral desde a medição do comprimento de seu estolão (6) até mesmo uso de quadrados vazados $(7)$, puçás $(8,9)$ ou bandejas $(10)$. Metodologias distintas podem levar a resultados distorcidos, errôneos e dificultam a comparação dos trabalhos.

O objetivo deste trabalho foi comparar as abundâncias de Odonata nas Lagoas do Camargo e Coqueiral utilizando duas metodologias de amostragem distintas, puçá e quadrado vazado, em duas espécies de macrófitas, E. aqurea e Salvinia auriculata (Aublet).

\section{Materiais e métodos}

As Lagoas do Camargo e Coqueiral estão localizadas na zona de desembocadura do Rio Paranapanema, na Represa de Jurumirim, SP (Figura 1). As duas lagoas estudadas, Camargo e Coqueiral, apresentam conexão com o Rio Paranapanema, entretanto apenas a Coqueiral possui conexão permanente com o rio.

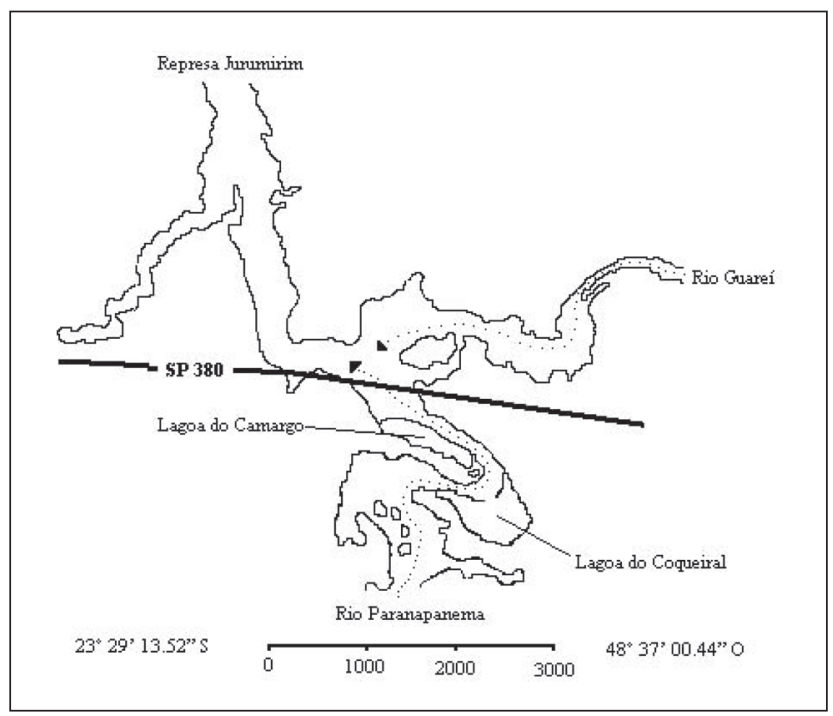

Figura 1 - Zona de transição entre a Represa de Jurumirim e o Rio Paranapanema e a localização das Lagoas do Camargo e Coqueiral 
Três bancos de E. azurea e três de $S$. auriculata foram selecionados nas Lagoas do Camargo e Coqueiral em julho de 2003. Foram realizadas três réplicas em cada ponto de amostragem para as macrófitas e para os fatores abióticos. Em cada estação de amostragem, foram medidos temperatura de superfície da água (com termístor Toho Dentam ${ }^{\circledR}$ ), oxigênio (11), $\mathrm{pH}$ (com pH-metro Micronal ${ }^{\mathbb{B}}$ B-380) e condutividade (com um condutivímetro HACH ${ }^{\circledR}$ modelo 2511) posteriormente corrigida a uma temperatura de $25{ }^{\circ} \mathrm{C}$ (11). As macrófitas foram amostradas com um puçá de formato quadrado com rede de $0,25 \mathrm{~mm}$ de malha e $0,09 \mathrm{~m}^{2}$ de área total e um quadrado vazado com área total de $0,225 \mathrm{~m}^{2}$. O puçá e o quadrado foram inseridos, respectivamente, sob e sobre o banco de macrófitas e apenas as que estavam no espaço amostral foram coletadas. Em laboratório, a fauna foi removida com movimentos circulares das plantas aquáticas em baldes contendo formol $8 \%$, formol $4 \%$ e água, respectivamente (10). O conteúdo do balde foi filtrado em peneira de $0,25 \mathrm{~mm}$ de malha e a fauna retida na peneira foi conservada em álcool 70\% até a triagem. A identificação dos odonatos foi realizada com bibliografia específica (12).

Para a comparação das abundâncias de Odonata entre o puçá e o método dos quadrados foi utilizado o test $t$ de Student. Uma análise de correlação de Spearman também foi realizada entre as abundâncias de Odonata e fatores abióticos.

\section{Resultados}

$\mathrm{Na}$ Figura 2, observamos na Lagoa do Camargo junto à E. azurea que as abundâncias de Telebasis sp. e Tauriphila sp foram menores quando amostradas com o método dos quadrados em relação ao puçá. Também observamos utilizando o quadrado vazado junto à $S$. auriculata uma redução na abundância de Telebasis sp.

$\mathrm{Na}$ Lagoa do Coqueiral junto à E. azurea e S. auriculata, utilizando o quadrado vazado notamos uma redução na abundância de quase todos os taxas amostrados em comparação ao puçá (Figura 3).

Observamos resultados distintos quanto à riqueza de Odonata entre as Lagoas do Camargo e Coqueiral. $\mathrm{Na}$ Camargo a riqueza foi maior quando utilizamos o método dos quadrados nas duas espécies de macrófitas analisadas (Figura 4). Já na Coqueiral, a riqueza foi maior com o puçá também nas duas espécies de plantas aquáticas (Figura 4).

$\mathrm{Na}$ Figura 5, pudemos observar que as abundâncias totais de Odonata foram sempre maiores com a utilização do puçá nas duas lagoas estudadas.

As maiores médias de oxigênio e temperatura de superfície da água foram observadas na Lagoa do Camargo (Figura 6). O pH e a condutividade mantiveram-se a 6,6 e $71 \mu \mathrm{S} . \mathrm{cm}^{-1}$, respectivamente, em todas as estações de amostragem nas Lagoas do Camargo e Coqueiral, exceto a condutividade junto à E. azurea na Lagoa do Coqueiral, onde registramos $73,5 \mu \mathrm{S} . \mathrm{cm}^{-1}$.

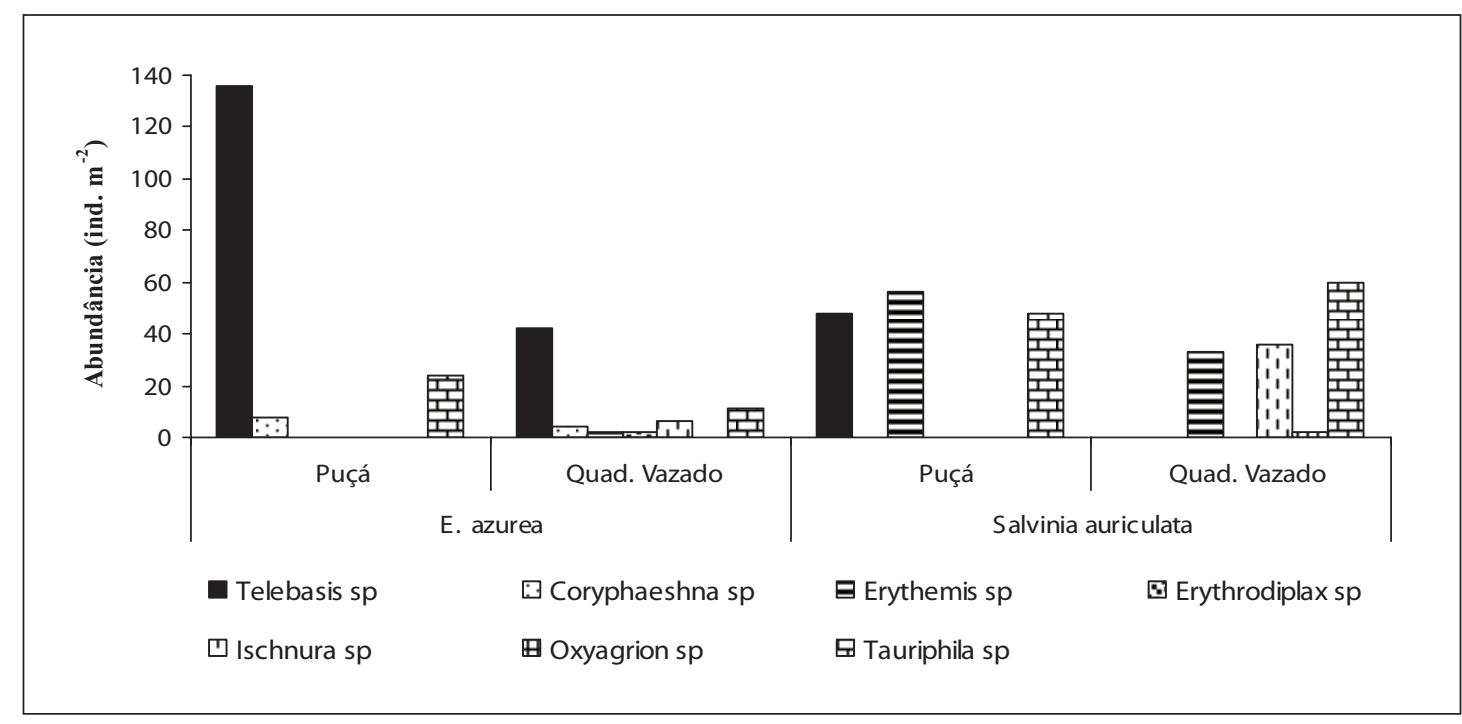

Figura 2 - Abundância absoluta (ind. $\mathrm{m}^{-2}$ ) dos gêneros de Odonata na Lagoa do Camargo junto à E. ažrea e $S$. auriculata com puçá e quadrado vazado 


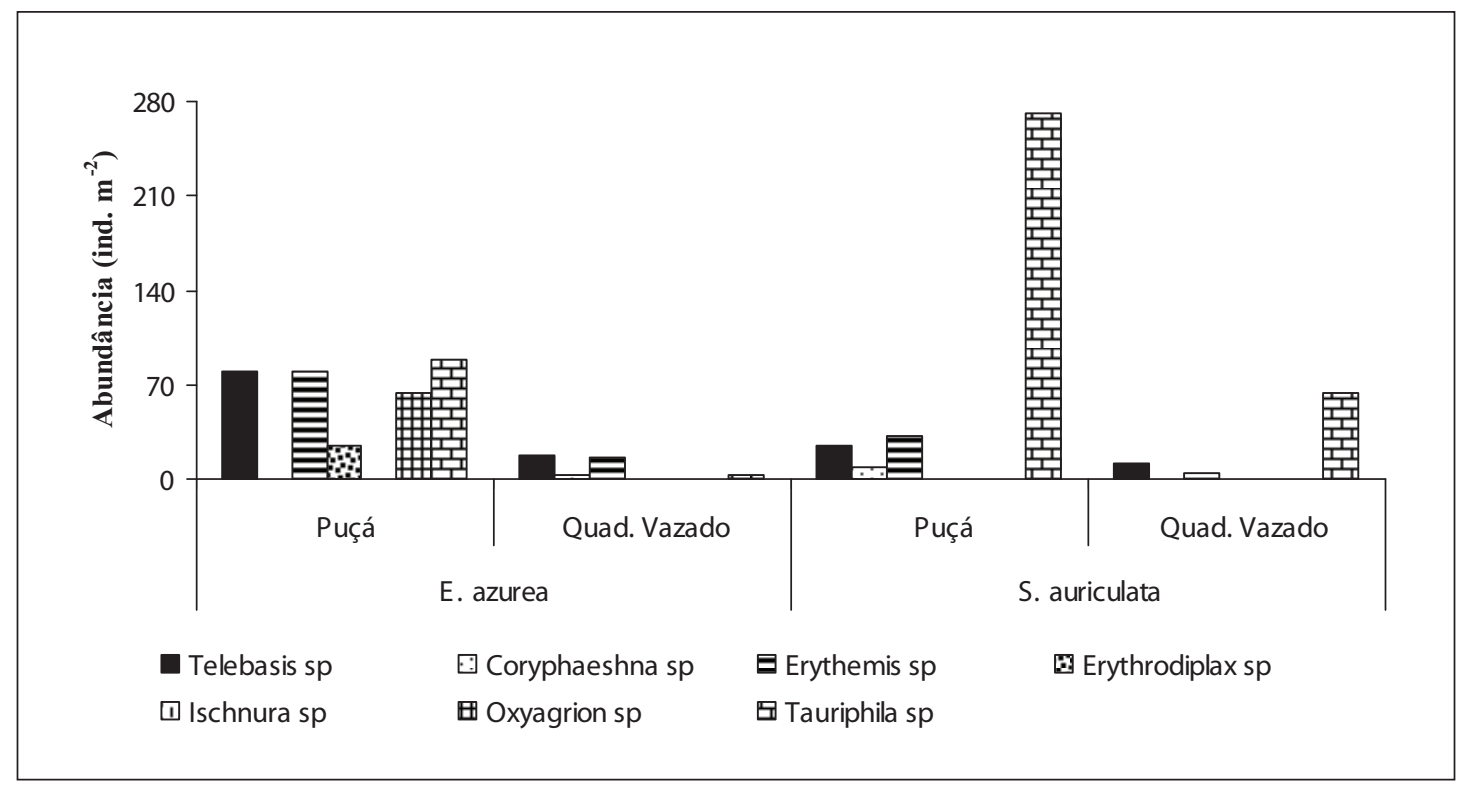

Figura 3 - Abundância absoluta (ind. $\mathrm{m}^{-2}$ ) dos gêneros de Odonata na Lagoa do Coqueiral junto à $E$. azurea e $S$. auriculata com puçá e quadrado vazado

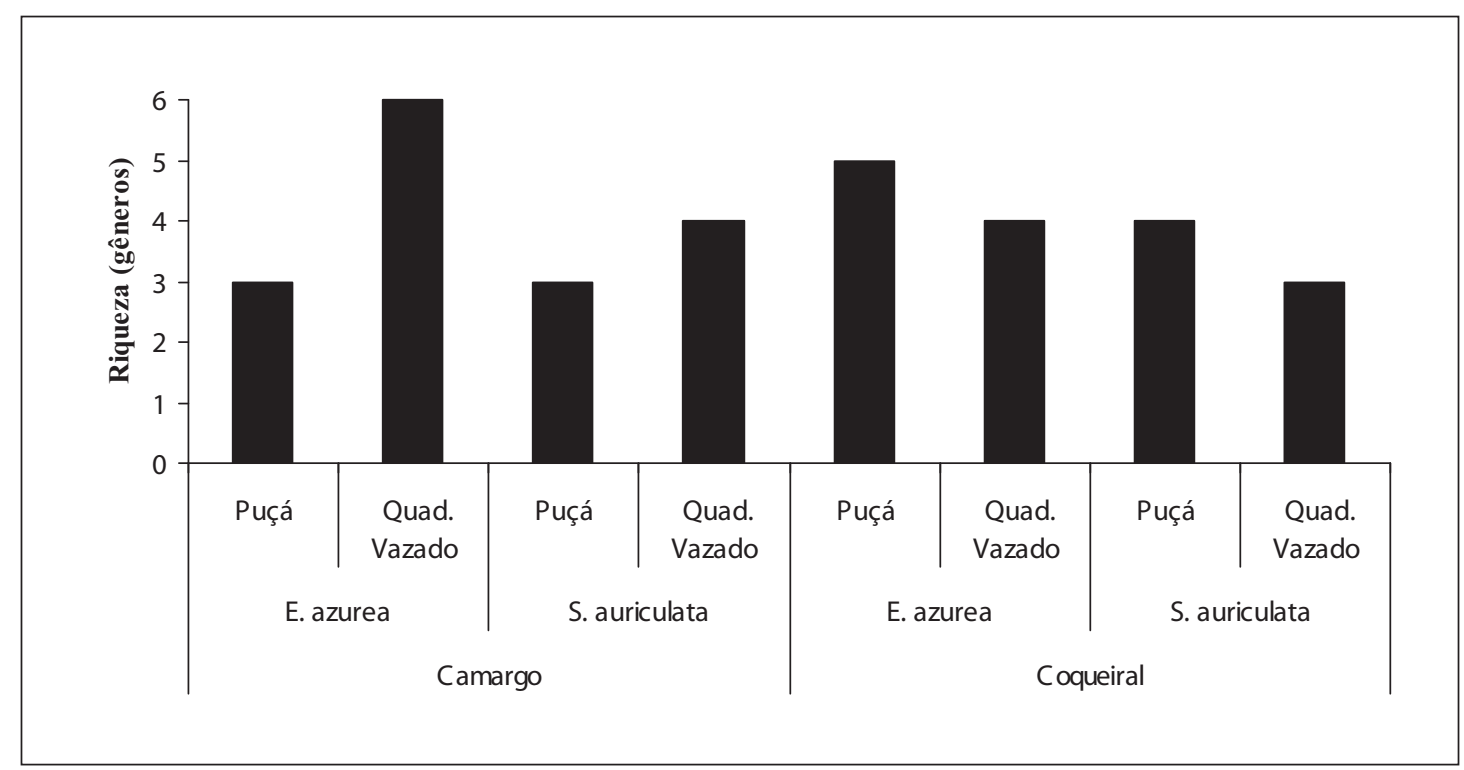

Figura 4 - Riqueza de gêneros de Odonata nas Lagoas do Camargo e Coqueiral junto à E. ažrea e $S$. auriculata com puçá e quadrado vazado

O teste $t$ de Student mostrou que não existe diferença significativa nas abundâncias de Odonata entre o puçá e o quadrado vazado. A correlação de Spearman também não mostrou correlação entre as abundâncias dos odonatos e fatores abióticos (oxigênio, $\mathrm{pH}$, condutividade e temperatura de superfície da água).

\section{Discussáo}

Segundo Corbet (4), ninfas de Odonata são predadoras vorazes que percebem suas presas a partir de pequenas vibrações na coluna de água. Segundo o autor, a capacidade de percepção dos imaturos de Odonata também pode ser utilizada 


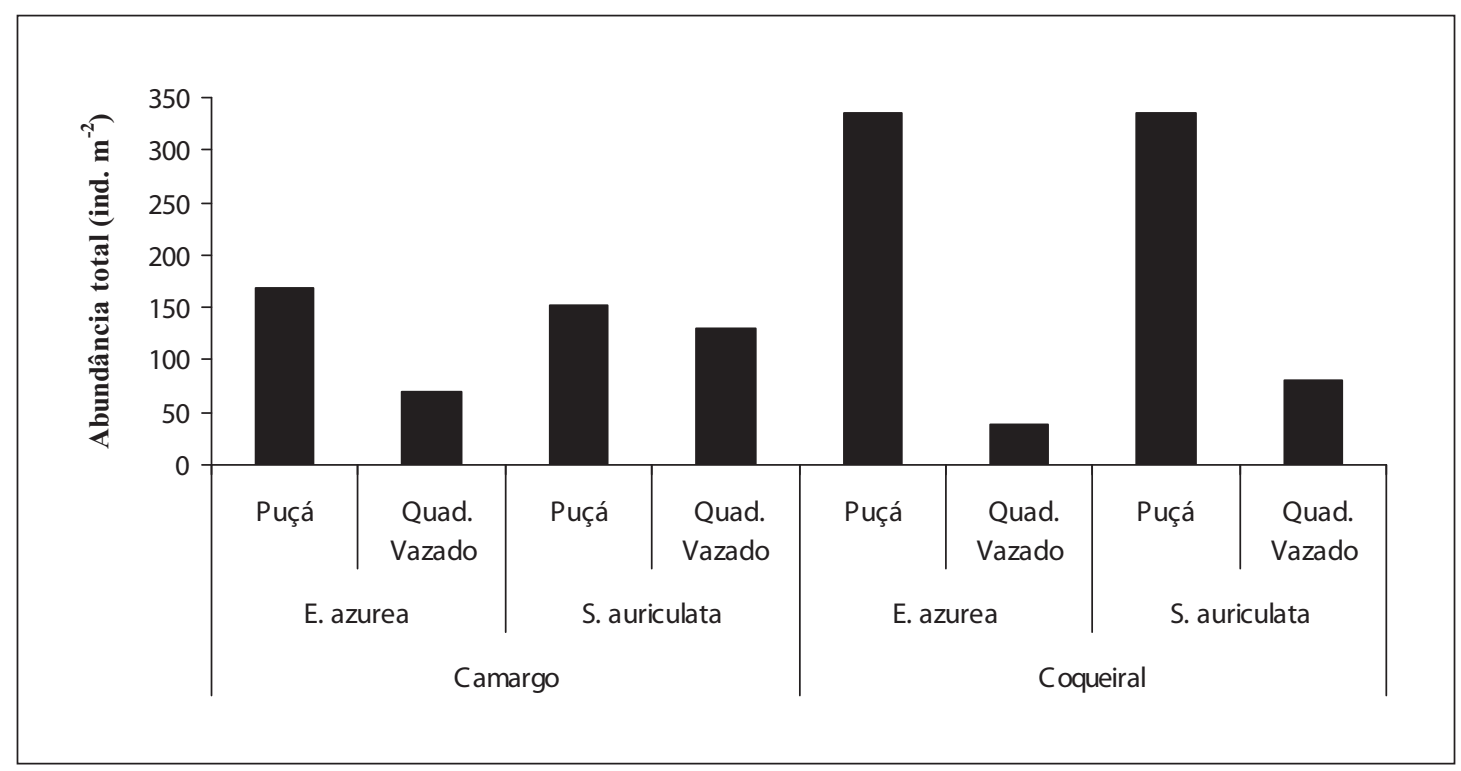

Figura 5 - Abundância total de Odonata (ind. $\mathrm{m}^{-2}$ ) nas Lagoas do Camargo e Coqueiral junto à E. a ąurea e $S$. auriculata com puçá e quadrado vazado

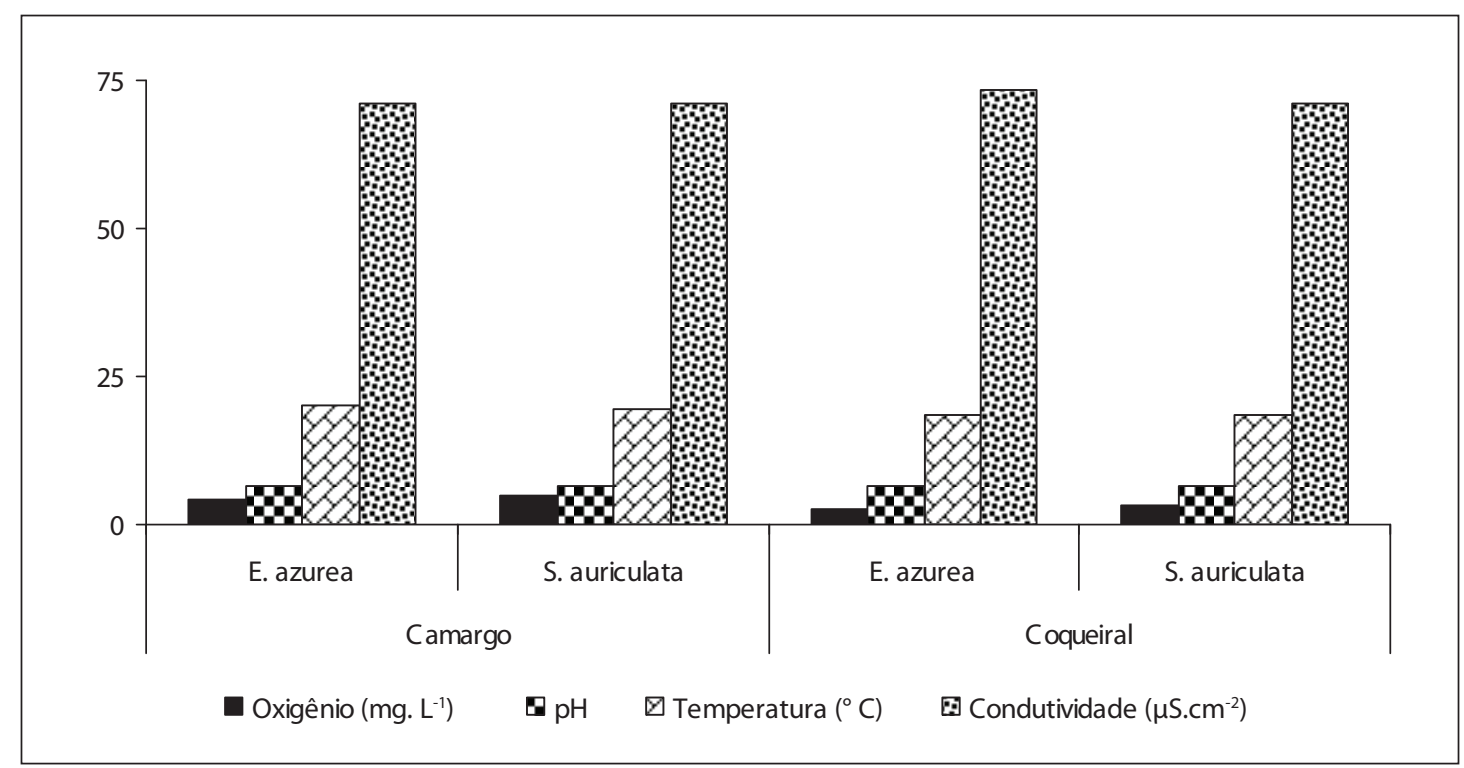

Figura 6 - Média de oxigênio $\left(\mathrm{mg} \cdot \mathrm{L}^{-1}\right), \mathrm{pH}$, temperatura $\left({ }^{\circ} \mathrm{C}\right)$ e condutividade $\left(\mu \mathrm{S} . \mathrm{cm}^{-1}\right)$ junto à E. azurea e $S$. auriculata nas Lagoas do Camargo e Coqueiral

como estratégia de fuga quando da aproximação de predadores como peixes. Em virtude da alta percepção das ninfas, era esperado que muitos imaturos fugissem pela simples manipulação das macrófitas, diminuindo sua abundância. Entretanto, a utilização de um puçá poderia minimizar a fuga das ninfas em relação ao quadrado vazado, graças à presença de uma rede que dificultava ou mesmo impedia a saída dos imaturos. Portanto, era esperada maior abundância total utilizando o puçá em comparação ao quadrado. Os resultados mostraram que em todo o trabalho as abundâncias totais foram sempre maiores utilizando o puçá em E. azurea e $S$. auriculata e nas Lagoas do Camargo e Coqueiral. Entretanto, a comparação das 
Fulan JA.

médias das abundâncias totais de Odonata não foi significativa. Apesar disso, as maiores abundâncias totais foram observadas na Lagoa do Coqueiral com o puçá. Diferenças quanto aos fatores abióticos entre as lagoas poderiam justificar maior abundância de Odonata com puçá na Coqueiral, porém a análise de correlação mostrou que o oxigênio, o pH, a temperatura e a condutividade não apresentaram correlação significativa com a abundância dos odonatos. Outros fatores, como a conectividade das lagoas estudadas com o rio, pode ter sido responsável pela maior abundância total de Odonata registrada na Lagoa do Coqueiral com o puçá.

Henry (13) destacou que as Lagoas do Camargo e Coqueiral apresentam diferentes graus de conexão com o Rio Paranapanema. Segundo o autor, a Camargo apresenta conexão temporária, enquanto a Coqueiral, conexão permanente. A Coqueiral é afetada diretamente pela velocidade da correnteza do rio, que atua diretamente sobre os padrões de distribuição da abundância de Odonata (14). Corbet (4) destacou que a influência da correnteza do rio pode dificultar a adesão das ninfas sobre as macrófitas e algumas espécies podem ser favorecidas por apresentarem comportamentos como agarrar ou esconder. Por outro lado, segundo o autor, a maior velocidade da correnteza também pode atuar, elevando a concentração de oxigênio, e, desta forma, aumentar a abundância de espécies características de ambientes lóticos, ou quando a correnteza for menor, reduzir a quantidade de oxigênio e aumentar a abundância de espécies de ambientes lênticos. Todos os gêneros registrados neste trabalho são característicos de ambientes lênticos (12). Portanto, uma concentração menor de oxigênio pode favorecer um aumento da abundância de gêneros de Odonata característicos de ambientes lênticos. Ao comparar-se a Lagoa do Camargo com a Coqueiral, notei que a quantidade de oxigênio foi maior na Coqueiral, que apresenta conexão com o rio. A menor concentração de oxigênio na Coqueiral não era esperada, pois, como apresenta conexão com o rio, a velocidade da correnteza poderia favorecer a maior quantidade de oxigênio. Entretanto, parece que a baixa concentração de oxigênio na Coqueiral favoreceu um aumento da abundância total de Odonata nesta lagoa, por apresentar apenas gêneros de Odonata característicos de ambientes lênticos. Além da concentração de oxigênio, a composição de gêneros observada na Coqueiral pode também ter influenciado a alta abundância com o puçá.
Ao se analisar a composição de gêneros de Odonata capturados com puçá na Lagoa do Coqueiral, notou-se que Telebasis sp., Tauriphila sp. e Erythemis sp. foram os grupos mais abundantes juntos à $S$. auriculata e E. azurea. Segundo Costa (12), Telebasis sp. possui um porte pequeno, Tauriphila sp. porte pequeno a médio, e Erythemis sp. porte médio. O porte pequeno a médio de Telebasis sp., Tauriphila sp. e Erythemis sp. possivelmente foi responsável pela alta abundância destes gêneros com o puçá, uma vez que este era revestido por uma rede que permitia a amostragem de ninfas menores. Este resultado fica evidente quando observamos Tauriphila sp. na Lagoa do Coqueiral junto E. azurea e $S$. auriculata. Observaram-se as maiores médias de abundância de Tauriphila sp. com o puçá na Lagoa do Coqueiral nas duas espécies de macrófitas. Além disso, Erythrodiplax sp. e Oxyagrion sp. foram praticamente só registradas com a utilização do puçá.

Em julho de 2004, juntoà E. ąureana Lagoa do Camargo e utilizando um puçá foi observado uma das menores riquezas e abundâncias de Odonata (15). Segundo os autores, três gêneros foram amostrados: Ishnura sp., Oxyagrion sp. e Telebasis sp.; este último foi o mais abundante no período. Quando se comparou a mesma Lagoa do Camargo utilizando a mesma metodologia de amostragem, o puçá, e a mesma espécie de macrófita, E. ąurea, observou-se que Telebasis sp. também foi o mais abundante; entretanto, não foi registrada a presença de Ishnura sp. e Oxyagrion sp. verificados em 2004. Em contrapartida, em julho de 2003 foram observados Tauriphila sp. e Coryphaeshna sp., que não foram amostrados em 2004. Quando se analisou em 2003 a amostragem realizada com o quadrado vazado, notou-se um resultado totalmente distinto do observado com o puçá; ou seja, com o quadrado foram registrados seis gêneros: Ishnura sp., Telebasis sp., Coryphaeshna sp., Erythemis sp., Erythrodiplax sp. e Tauriphila sp. Portanto, se fossem comparados os resultados da riqueza com o puçá entre os anos de 2003 e 2004, se concluiria que não houve diferença na riqueza de gêneros de Odonata; entretanto, se comparássemos os resultados utilizando o quadrado, observaríamos uma redução de 50\% na riqueza em 2004 em relação a 2003. Neste caso, o método de amostragem poderia ter afetado diretamente os resultados e permitiria conclusões que não corresponderiam à realidade.

Em resumo, não houve diferenças significativas entre as amostragens com quadrado vazado 
e puçá em E. azurea e $S$. auriculata nas Lagoas do Camargo e Coqueiral. Entretanto, o puçá se mostrou mais eficiente na captura de ninfas de tamanho menor a médio, como Telebasis sp., Tauriphila sp. e Erythemis sp.

\section{Referências}

1. Esteves FA. Fundamentos de limnologia. Rio de Janeiro: Interciência; 1998.

2. Weatherhead MA, James MR. Distribution of macroinvertebrates in relation to physical and biological variables in the littoral zone of nine New Zealand lakes. Hydrobiologia. 2001;462:115-29.

3. Capitulo AR. Los Odonata de la República Argentina (Insecta): fauna de agua dulce de la República Argentina. Profadu (Conicet). 1992;34(1):1-91.

4. Corbet PS. Dragonflies: behavior and ecology. New York: Cornell University Press; 1999.

5. Butler RG, Maynadier PG. The significance of littoral and shoreline habitat integrity to the conservation of lacustrine damselflies (Odonata). Journal of Insect Conservation. 2008;12:23-36.

6. Monkolski A, Takeda AM, Melo SM. Fauna structure of water mites associated with Eichhornia azurea in two lakes of the upper Paraná floodplain, Mato Grosso do Sul State, Brazil. Acta Scientarium Biological Sciences. 2005;27(4):329-37.

7. Moretti MS, Goulart MDC, Callisto M. Avaliação rápida da macrofauna associada a Eichhornia azurea (Swartz) Kunth, 1843 e Pontederia lanceolata Nutt., 1818 (Pontederiaceae) na Baía do Coqueiro, Pantanal de Poconé (MT/Brasil). Revista Brasileira de Zoociências. 2003;5(1):7-22.

8. FulanJA, Henry R. Distribuição temporal de imaturos de Odonata (Insecta) associados à Eichhornia azurea (Kunth) na Lagoa do Camargo, Rio Paranapanema, São Paulo. Revista Brasileira de Entomologia. 2007;51(2):224-7.
9. Rocha-Ramirez A, Ramirez-Rojas A, Chavez-Lopez $\mathrm{R}$, Alcocer J. Invertebrate assemblages associated with root masses of Eichhornia crassipes (Mart.) SolmsLaubach 1883 in the Alvarado Lagoonal System, Veracruz, Mexico. Aquatic Ecology. 2007;41:319-33.

10. Afonso AAO. Relações da fauna associada à Eichhornia azurea (Swartz) Kunth com as variáveis abióticas em lagoas laterais de diferentes graus de conexão ao Rio Paranapanema (zona de desembocadura na Represa de Jurumirim, SP) [tese]. Botucatu: Universidade Estadual Paulista; 2002.

11. Golterman KL, Clymo RS, Ohmstad MAM. Methods for physical and chemical analysis of freshwaters. Londres: Blackwell Scientific Publication; 1978.

12. Costa JM, Souza LOI, Lourenço NA, Oldrini BB. Chave para identificação das famílias e gêneros das larvas conhecidas de Odonata do Brasil: comentários e registros bibliográficos. Publicações Avulsas do Museu Nacional do Rio de Janeiro. 2004;99:3-42.

13. Henry R. The connectivity of the Paranapanema river with two lateral lakes in its mouth zone into the Jurumirim Reservoir. Acta Limnologica Brasiliensis. 2005;17(1):57-69.

14. Hofmann TA, Mason CF. Habitat characteristics and the distribution of Odonata in a lowland river catchment in eastern England. Hydrobiologia. 2005;539(1):137-47.

15. Fulan JA, Henry R. The Odonata (Insecta) assemblage on Eichhornia azurea (Sw.) Kunth (Pontederiaceae) stands in Camargo Lake, a lateral lake on the Paranapanema River (State of São Paulo, Brazil), after an extreme inundation episode. Acta Limnologica Brasiliensia. 2006;18(4):423-31.

Recebido: 10/10/2008 Received: 10/10/2008

Aprovado: 15/11/2009 Approved: 11/15/2009 